\title{
Magneto-optical evidence of the percolation nature of the metal-insulator transition in the two-dimensional electron system
}

\author{
I. V. Kukushkin \\ Max-Planck-Institut für Festkörperforschung, Heisenbergstrasse 1, 70569 Stuttgart, Germany \\ and Institute of Solid State Physics, Russian Academy of Sciences (RAS), Chernogolovka, 142432 Russia \\ Vladimir I. Fal'ko \\ School of Physics and Chemistry, Lancaster University, LA1 4YB, Lancaster, United Kingdom; \\ Institute of Solid State Physics, Russian Academy of Sciences (RAS), Chernogolovka, 142432 Russia; \\ and Max-Planck-Institut für Festkörperforschung, Heisenbergstrasse 1, 70569 Stuttgart, Germany \\ R. J. Haug \\ Max-Planck-Institut für Festkörperforschung, Heisenbergstrasse 1, 70569 Stuttgart, Germany \\ and Institut für Festkörperphysik, Universität Hannover, Appelstrasse 2, 30167 Hannover, Germany \\ K. v. Klitzing and K. Eberl \\ Max-Planck-Institut für Festkörperforschung, Heisenbergstrasse 1, 70569 Stuttgart, Germany \\ (Received 9 February 1996; revised manuscript received 2 April 1996)
}

\begin{abstract}
We compare the results of the transport and time-resolved magnetoluminescence measurements in disordered two-dimensional (2D) electron systems in $\mathrm{GaAs}_{-} \mathrm{Al}_{x} \mathrm{Ga}_{1-x} \mathrm{As}$ heterostructures in the extreme quantum limit, particularly in the vicinity of the metal-insulator transition (MIT). At filling factors $\nu<1$, the optical signal has two components: the single-rate exponentially decaying part attributed to a uniform liquid and a power-law long-living tail specific to a microscopically inhomogeneous state of electrons. We interpret this result as a separation of the $2 \mathrm{D}$ electron system into liquid and localized phases, especially because the MIT occurs strikingly close to those filling factors where the liquid occupies $\frac{1}{2}$ of the sample area (the percolation threshold condition in two-component media). [S0163-1829(96)52320-9]
\end{abstract}

The metal-insulator transition (MIT) in two-dimensional (2D) electron systems continues to be a hot topic in semiconductor physics, ${ }^{1-3}$ particularly because of a variety of transitions between quantum Hall liquids and insulating states in the extreme quantum limit realized at high magnetic fields. Depending on the level of disorder in the system, the MIT can be due either to the Wigner crystallization or to localization. In pure enough systems, it has the thermodynamic character and is conventionally attributed to the interaction-induced crystallization of 2D electrons into the Wigner solid. ${ }^{3}$ In disordered structures, the dielectric state is formed because of localization of the $2 \mathrm{D}$ electrons by a random potential coming from an inhomogeneous charge distribution in the donor layer, ${ }^{1,2}$ and the interest of the present publication is focused on the latter situation.

Although the single-particle scenario of localization of electrons at high magnetic fields was already thoroughly explored some years ago, ${ }^{4}$ the most recent theories ${ }^{5}$ emphasize the influence of interactions on the MIT also in disordered systems, especially regarding the existence of a hierarchy of strongly correlated quantum Hall liquids. Being inspired by the success of the scaling ideas developed in single-particle models, ${ }^{4}$ the search of a global phase diagram of the MIT in the extreme quantum limit has been started ${ }^{5}$ in order to describe the growing amount of experimental data. However, one should not ignore the possibility that, in interacting electron systems, the transiton between correlated liquids and disordered insulator is the Mott transition rather than the
Anderson one, and that the mesoscopic-scale inhomogeneity in the strength of a random potential itself can result in the separation of the 2D system into phases with locally different properties (liquid versus localized insulator). In this scenario, the portion of a localized phase varies continuously under the variation of a magnetic field due to the sensitivity of the electron-electron correlations to the value of the filling factor, so that the MIT detected in the transport might manifest nothing but the destruction of the percolation through the liquid in a multicomponent medium. ${ }^{6}$

To find the truth, it is necessary to make a local measurement that would distinguish between different phases (liquid and insulator) and to evaluate the amount of each of them. We solve this problem by using the method based on the analysis of the time-resolved recombination of $2 \mathrm{D}$ electrons with a small number of acceptor bound holes in the $\delta$-doped layer. We already know $^{7}$ that, in contrast to the quantum Hall effect state at the filling factor $\nu=1$ that demonstrates the single-exponential decay $I(t) \propto \exp \left(-t / t_{0}\right)$, the magnetically frozen state manifests itself in a broad spectrum of recombination rates, which results in the power-law kinetics of the magnetoluminescence. It is a peculiarity of the recombination process involving acceptor bound holes that the latter form small neutral centers, and that the lifetime $\tau$ of each hole is determined by the local density of $2 \mathrm{D}$ electrons at the acceptor position, $\tau^{-1} \propto \rho_{e}$, so that the spectrum $f\left(\tau^{-1}\right)$ of recombination rates $\tau^{-1}$ reflects the distribution of the local 2D electron density. ${ }^{8}$ The latter can be found from 
TABLE I. Sample parameters: density $n_{e}$, mobility $\mu, t_{0}$.

\begin{tabular}{lcccccc}
\hline \hline \multicolumn{1}{c}{ Sample } & A & B & C & D & E & F \\
\hline$n_{e}\left(\times 10^{11} \mathrm{~cm}^{-2}\right)$ & 0.55 & 0.60 & 0.80 & 0.71 & 0.60 & 1.43 \\
$\mu\left(\times 10^{5} \mathrm{~cm}^{2} / \mathrm{V} \mathrm{s}\right)$ & 12.1 & 3.85 & 0.94 & 0.43 & 0.21 & 0.11 \\
$t_{0}\left(\times 10^{-7} \mathrm{~s}\right)$ & 1.9 & 1.9 & 2.3 & 2.2 & 2.0 & 2.9 \\
\hline \hline
\end{tabular}

the inverse Laplace transform of the measured temporal dependence $I(t)$ of the luminescence intensity after a short illumination pulse, $I(t) \propto \int d \tau^{-1} f\left(\tau^{-1}\right) e^{-t / \tau}$. For example, the above-mentioned single-exponential decay of the luminescence at $\nu=1$ evidences the microscopic-scale homogeneity of the $2 \mathrm{D}$ electron density in this incompressible liquid state, ${ }^{11}$ whereas the information about the distribution of local values $\rho_{e}$ that we extract from the long-living tails of luminescence from any state at $\nu<1$ may be used for identification of its intrinsic structure, as has been done before in the Wigner solid regime. ${ }^{7}$

In the present paper we combine the transport measurements with the time-resolved optics ${ }^{7,9}$ to study systematically a series of heterostructures with various densities and levels of disorder. In the entire interval of filling factors $\nu<1$ and in all the samples, the magnetoluminescence kinetics $I(t)$ looks as if it is composed of a single-exponential (liquid-type) contribution $I_{l}(t) \propto \exp \left(-t / t_{0}\right)$ and the universal contribution $I_{i}(t)$ from inhomogeneous localized state with power-law long-living asymptotics $I_{i}(t) \propto\left(t_{0} / t\right)^{\alpha}$ summed with different weights. This enables us to distinguish between the liquid and localized phases and to estimate their portions in the mixture. Our result shows that for all the samples under investigation the liquid phase fills about $\frac{1}{2}$ of the sample area at the MIT point, which coincides with the condition of the percolation threshold in 2D two-component media. $^{6}$

We studied six different-quality (see Table I) GaAs$\mathrm{Al}_{x} \mathrm{Ga}_{1-x} \mathrm{As}$ single heterojunctions with a $\delta$-doped monolayer of Be acceptors $\left(n_{A}=1.3 \times 10^{9} \mathrm{~cm}^{-2}\right)$ located in the wide $(1 \mu \mathrm{m}) \mathrm{GaAs}$ buffer layer at a distance about $30 \mathrm{~nm}$ from the interface. ${ }^{10}$ The magnetoresistance tensor was measured on the Hall bar samples by use of a standard lowfrequency $(12 \mathrm{~Hz})$ and low-current $(10 \mathrm{nA})$ lock-in technique with a resistance of $100 \mathrm{M} \Omega$ connected in series with the sample. For photoexcitation, we used pulses from a tunable Ti-sapphire laser (wavelength $\sim 800 \mathrm{~nm}$ ) with a duration of $20 \mathrm{~ns}$, peak power of $10^{-5}-10^{-4} \mathrm{~W} / \mathrm{cm}^{2}$, and frequency of $10^{5}-10^{3} \mathrm{~Hz} .70$

Figure 1 shows the results of magnetotransport measurements (in the range of temperatures $T=50-150 \mathrm{mK}$ ) that were used for determining the MIT point in two samples $B$ and $E$, which have identical concentrations but very different mobilities (see Table I). The conductivity measured in samples of different qualities shows quite distinguishable behavior, especially at $\nu<1$. In particular, in better-quality samples $(A, B, C)$ one can find the intercrossing between $\sigma_{x x}(B)$ taken at different temperatures, which can be used for determination of the MIT point. ${ }^{1}$ However, such an intercrossing does not appear in the magnetoconductivity data taken in the lowest mobility structures $(D, E, F)$, so that another criterion of the MIT is desirable. The common feature of all studied samples near the MIT was an abrupt appear-
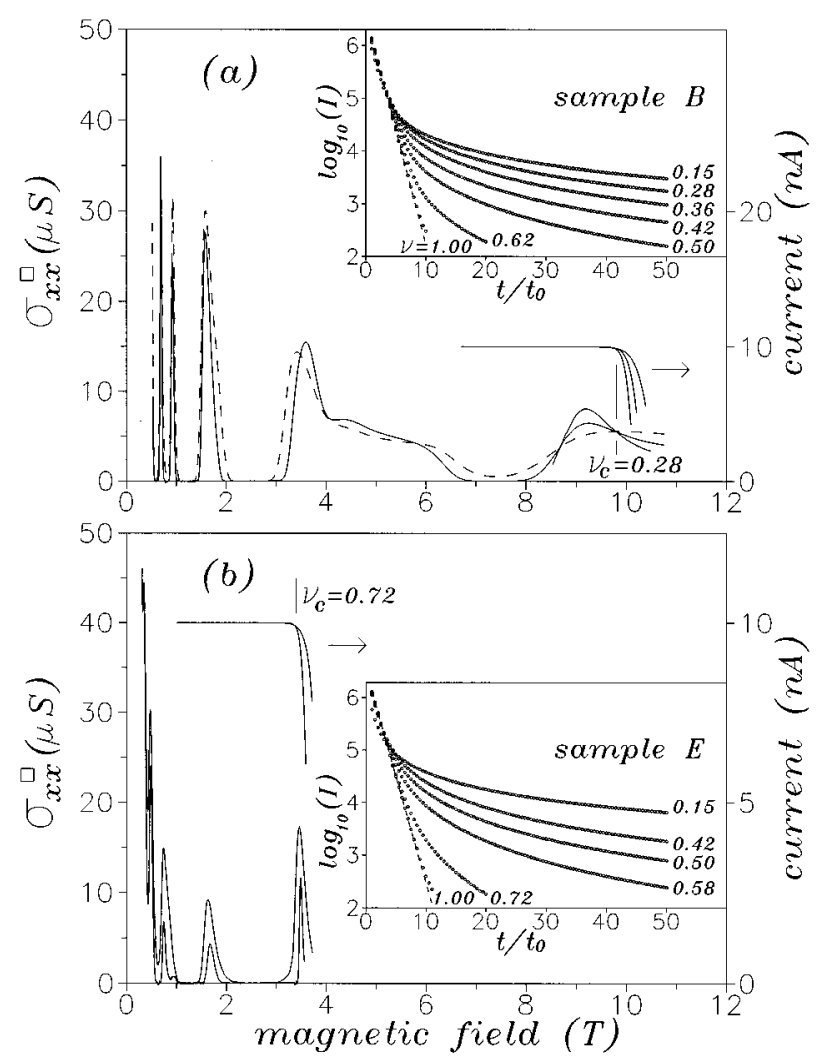

FIG. 1. Magnetic-field dependence of the conductivity and current measured in samples $B$ (a) and $E$ (b) for different temperatures [(a) $-T=150,100,50 \mathrm{mK}$, and (b) $-T=100,50 \mathrm{mK}]$. The critical filling factor $\nu_{c}$ of the MIT is indicated. Insets represent the raw data of the timedependent magnetoluminescence kinetics measured in the same samples at filling factors $\nu<1$ at $T=50 \mathrm{mK}$.

ance of the out-of-phase signal both in the diagonal and Hall components of resistivity, which is accompanied by a drastic decrease of a current and indicates the formation of a dielectric state. Figure 1 also shows the evolution of the magneticfield dependence of the current through the $2 \mathrm{D}$ system with the temperature. Under the decrease of filling factor, the current shows a threshold at $\nu_{c}$, which becomes more pronounced at lower temperatures, and is treated below as the indication of the MIT point. The value of $\nu_{c}$ determined in such a way is fairly close to the position of the peak in $\sigma_{x x}$ in samples $D-F$, which has been used by Glozman et al. and others $^{2}$ as a criterion of MIT. Note that in the present investigation we do not fight for the accuracy of determining $\nu_{c}$ better than a few percent, since our aim is to see a correspondence between the MIT data observed in transport and optical data that show no threshold behavior. Indeed, in contrast to the transport, the optical data taken in the same samples demonstrate only a continuous evolution both of the integral and time-resolved spectra. Insets to Figs. 1(a) and 1(b) show the raw data of typical recombination kinetics measured in samples $(B, E)$ in different magnetic fields at $T=50 \mathrm{mK}$ and we see no critical changes occuring when $\nu=\nu_{c}$ is passed.

The magnetoluminescence kinetics measured in lowerand higher-mobility samples has both common features and significant differences. The common feature of the recombination kinetics observed in all the samples is the purely exponential decay, $I(t) \propto \exp \left(-t / t_{0}\right)$, of the magnetoluminescence intensity at the filling factor $\nu=1$. Since the 


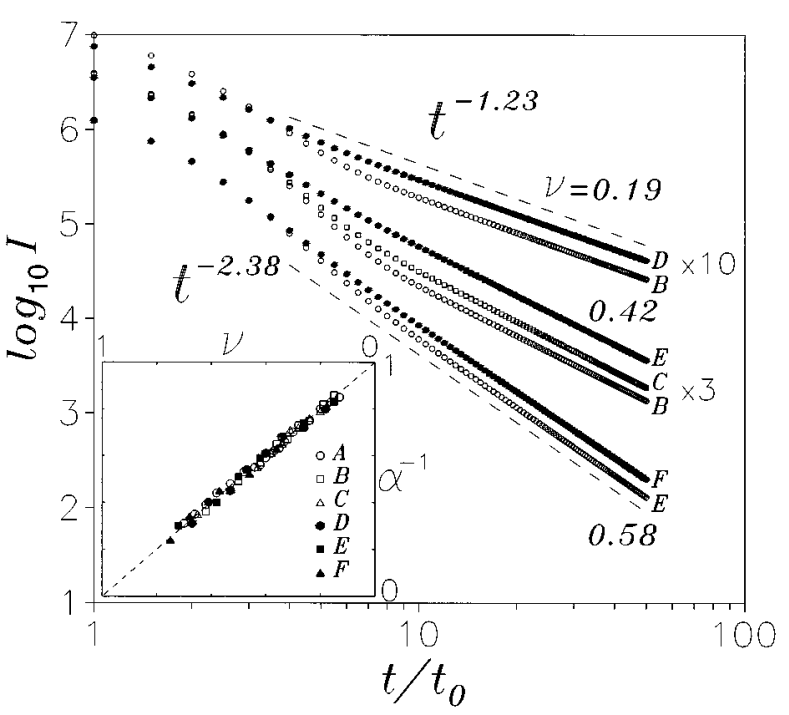

FIG. 2. Comparison of the recombination kinetics in groups of samples taken at three different filling factors and at $T=50$ $\mathrm{mK}$. The integral luminescence intensity is normalized by the same value within each group. Dashed lines illustrate a draft fit of the long-living tail using a power law. Inset shows the dependence of $\alpha^{-1}$ on filling factor measured in all the studied samples.

density of optically active holes is much smaller than the 2D electron density, the time dependence $I(t)$ is controlled by the recombination rates of holes. In the homogeneous electron system, the latter are determined by the sheet electron density and the distance from the interface to the acceptor monolayer and are the same for all of them, so that the single-rate $\left(\tau^{-1}=t_{0}^{-1}\right)$ exponential decay manifests the microscopic-scale homogeneity of the electron density. At $\nu=1$, this is the well-known property of a trial many-body wave function corresponding to a completely filled Landau level. ${ }^{11}$

For the lower fillings, the slow recombination tail develops, which indicates the formation of a short-range-scale inhomogeneity of the local electron density (on the scale of $n_{e}^{-1 / 2}$ ). This behavior is common for all samples we studied and evidences a partial localization of the system under an increase of a magnetic field that starts much earlier than the MIT takes place. Here, the difference between low- and high-mobility samples manifests itself: in the former, the long-living tails develop at considerably higher filling factors (lower magnetic fields). To make a comparison easier, in Fig. 2 we show the kinetics of magnetoluminescence $I(t)$ for three groups of samples $(\{B, D\},\{B, C, E\},\{E, F\})$, each group being studied at the same filling factor $(\nu=0.19,0.42,0.58)$. Within each group of samples, the luminescence intensity presented in Fig. 2 is normalized to the same integral value [e.g., $\int I^{(B)}(t) d t=\int I^{(D)}(t) d t$ in the first group], which enables us to compare both the form of the long-living tails and their strength in samples with different parameters and at the same or different values of the filling factor.

First of all, after comparing the data taken in different samples at the same $\nu$, to, say, $B, C$, and $E$ for $\nu=0.42$, we find that the strength of the long-living recombination tail is systematically more significant in lower-quality samples. At the same time, the form of the tails at a long enough delay time is essentially the same for different samples studied at the same values of the filling factor. The presentation of the data in Fig. 2 using the log-log plot has been chosen in order to demonstrate that the recombination kinetics at the longliving tails obeys the power law, ${ }^{7,9}$

$$
I(t)=A\left(t_{0} / t\right)^{\alpha}, \quad t>t_{0},
$$

and that the exponents $\alpha$ of the power-law dependence coincide in different samples, if we make the measurement at the same filling factor, although the strength of the tail (i.e., parameter $A$ ) is higher when disorder is stronger. The powerlaw kinetics has been predicted by the earlier theory of recombination in the extreme quantum limit, ${ }^{8}$ with the exponent $\alpha$, which can be approximated by $\alpha \approx 1+\nu$ at $\nu \ll 1$. The fact that the exponent of the magnetoluminescence kinetics calculated in Ref. 8 depends only on the value of the filling factor was the result of the assumption that in the magnetically frozen state electrons are strongly localized at the interparticle distances-each sitting opposite its own donor behind a narrow spacer. The spectrum of experimentally derived $\alpha$ 's for all the available samples and for a broad range of filling factors, $0.75>\nu>0.15$, is presented in the inset to Fig. 2. Although these data correspond to the samples of a very different quality and concentration, the experimentally obtained values of an $\alpha$ group near a single filling factor dependence $\alpha(\nu)$. This is what we call the universality of the power-law recombination kinetics of localized electrons. In a good approximation, the empirical $\alpha$ has the form $\alpha \approx(1-\nu)^{-1}$, which is consistent with the theory by $\mathrm{Fal}^{\prime} \mathrm{ko}^{8}$ for $\nu \ll 1$.

As we discussed above, the kinetics of recombination of $2 \mathrm{D}$ electrons with acceptor-bound holes in a $\delta$-doped layer is governed by the spectrum of the recombination rates of holes, $\tau^{-1} \propto \rho_{e}$, which, in its turn, reflects the character of the distribution $f\left(\rho_{e}\right)$ of the local 2D electron density, $\rho_{e}$. From this point of view, the observed power-law kinetics of recombination in Eq. (1) can be directly recalculated (using the inverse Laplace transform) into the power-law distribution of the local 2D electron density, $f\left(\rho_{e}\right) \propto \rho_{e}^{\beta}$ with $\beta=\alpha-2 \approx(2 \nu-1) /(1-\nu)$ and the observed universality of the luminescence kinetics has behind it the universality of the distribution of a local density in a localized disordered state of magnetically frozen electrons. Below, we use this fact for measuring the disorder-dependent portions of insulating and liquid phases in the sample. Figure 3 shows that the extraction of the power-law tail from the total intensity gives the rest of it, which has a typical single-exponential behavior at the intermediate range of the delay time. The parameters ( $A$ and $\alpha$ ) of the slow recombination tail $I_{i}(t)=A\left(t_{0} / t\right)^{\alpha}$ were determined from a fit of the power-law asymptotics at $t \gg t_{0}$. The rest of the recombination intensity, $I_{l}=I(t)-I_{i}(t)$, is represented in Fig. 3 by solid circles and demonstrates an exponential decay, $I_{l}(t) \propto \exp \left(-t / t_{0}\right)$, which can be followed over the same number of decades as at $\nu=1$. The single-rate decay is the property of a microscopically homogeneous density, so that we assign this part of the luminescence intensity to the liquid state of electrons and suggest that the 2D electron system represents a mixture of a liquid and disorder-induced localized phases.

The goal of our analysis is to construct a diagram illustrating the evolution of the portion of the sample area, 


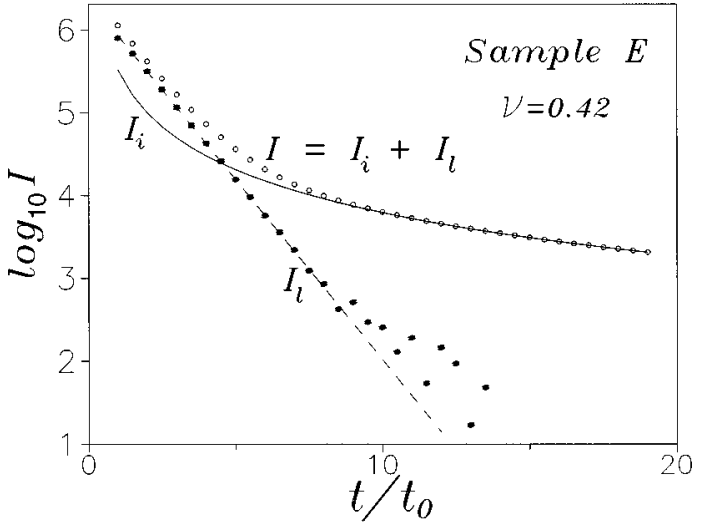

FIG. 3. Example of a separation of the time-dependent magnetoluminescence intensity $I(t)$ into the power-law and exponential parts, $I=I_{i}+I_{l}$. Solid line corresponds to the fit of the long-living tail of $I(t)$ by the power law $I_{i}(t)=A\left(t_{0} / t\right)^{\alpha(\nu)}$. Solid circles and dashed line are the dependence $I_{l}(t)=I(t)-I_{i}(t)$ at an interemediate range of the delay time and a single-exponential fit to it

$S_{l}(\nu)=\left[\int I_{l} d t\right] /\left[\int I d t\right]$, occupied by a liquid under the decrease of the filling factor. The dependence $S_{l}(\nu)$ determined for six different samples is shown in Fig. 4. The relative amount of the liquid phase in all the structures tends to decrease under an increase of a magnetic field, which manifests the formation of the freeze-out regime. In the low-mobility samples $(D, E, F)$, the localization is much more efficient and develops smoothly in the entire region of filling factors $\nu<1$.

In the better-quality samples $(A, B, C)$, the general tendency of the portion of the liquid to decrease with increasing of a magnetic field is accompanied by oscillations associated with the fractional quantum Hall effect states (see Fig. 4). The amount of the recombination events that we assign to the liquid has clear maxima at $\nu=\frac{1}{3}$ in samples $B$ and $C$, and at $\frac{1}{3}, \frac{1}{5}, \frac{2}{5}$ in sample $A$. This is a manifestation of the incompressibility of the Laughlin liquid, in which the interaction effects are strong enough (compared to disorder) to keep the electron density microscopically homogeneous. ${ }^{11}$ This supplies us with an additional argument in favor of the assumption that the separation of the system into the liquid and insulating phases at any filling factor is the result of a competition between electron-electron interactions and disorder, in which the former attempt to form a homogeneous liquid state whereas the latter tends to break correlations among electrons and to localize them. Note that even the best-

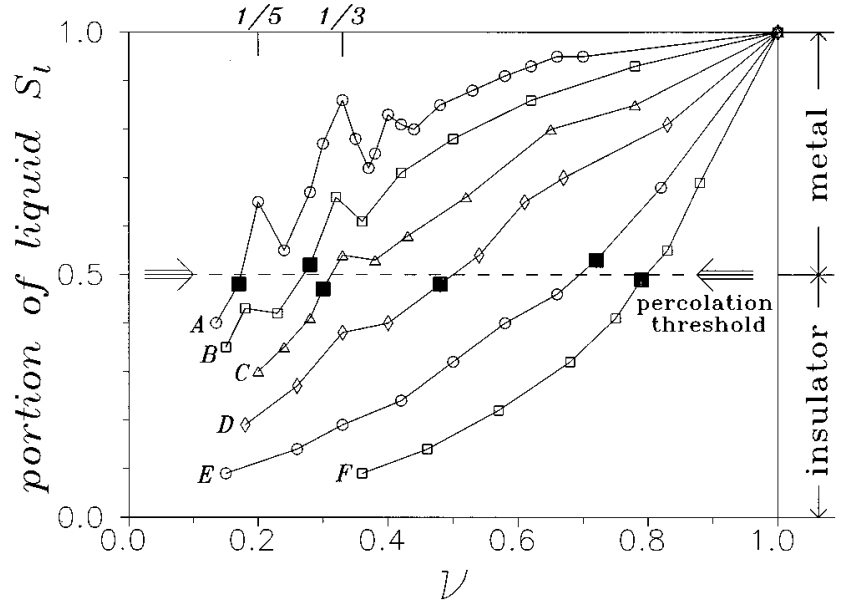

FIG. 4. The filling factor dependence of a portion of the sample area occupied by a liquid in all studied structures. Black squares indicate the points taken exactly at the filling factors of the MIT detected in the transport measurements. Dashed line and arrows illustrate the classical condition of a percolation threshold in two-component systems mixed of a metallic and insulating phases.

quality samples studied in the present paper were disordered enough and did not show abrupt features specific to the Wigner crystallization. ${ }^{7}$

Let us finally focus on the region near the critical filling factor $\nu_{c}$ of the MIT determined using the transport measurements (see Fig. 1). For each single sample, the optically characterized level of localization smoothly changes near $\nu_{c}$ and shows no striking features. However, being compared in a wide variety of structures, the MIT always occurs strikingly close to those filling factors where the continuously varying portion of a liquid fills one half of the sample area. The fundamental property of the percolation transition in macroscopically inhomogeneous 2D media is that it occurs when the insulating component occupies one half of the sample area; that is when the percolation path through the conducting phase is destroyed. ${ }^{6}$ Our conclusion is that the magnetic-field-driven MIT that we observe at $\nu<1$ is related to the percolation threshold through the liquid in the systems with the phase separation into a liquid and disorder insulator.

The authors thank Volkswagen Stiftung and the Russian Fund of Fundamental Research for financial support. One of us (V.F.) also acknowledges support from EPSRC and NATO CRG 921333.
${ }^{1}$ H. W. Jiang et al., Phys. Rev. Lett. 65, 633 (1990); H. W. Jiang et al., Physica B 197, 449 (1994); R. Hughes et al., J. Phys. Condens. Matter 6, 4763 (1994).

${ }^{2}$ I. Glozman et al., Phys. Rev. Lett. 74, 594 (1995); D. Shahar et al., ibid., 74, 4511 (1995).

${ }^{3}$ E. Y. Andrey et al., Phys. Rev. Lett. 60, 2765 (1988).

${ }^{4}$ D. E. Khmelnitskii, Pis'ma Zh. Eksp. Teor. Fiz. 38, 454 (1983) [JETP Lett. 38, 552 (1983)]; H. Levine et al., Phys. Rev. Lett. 51, 1915 (1983); R. Laughlin, ibid. 52, 2304 (1984).

${ }^{5}$ A. Karlhede et al., in Correlated Electron Systems, edited by V. J. Emery (World Scientific, Singapore, 1992), p. 242; S. Kivel- son et al., Phys. Rev. B 46, 2223 (1992).

${ }^{6}$ A. L. Efros and B. I. Shklovskii, Electronic Properties of Doped Semiconductors (Springer, Berlin, 1984), p. 107.

${ }^{7}$ I. V. Kukushkin et al., Phys. Rev. Lett. 72, 3594 (1994).

${ }^{8}$ V. I. Fal'ko, Phys. Rev. B 49, 2242 (1994).

${ }^{9}$ I. V. Kukushkin et al., Pis'ma Zh. Eksp. Teor. Fiz. 63, 118 (1996) [JETP Lett. 63, 132 (1996)].

${ }^{10}$ I. V. Kukushkin et al., Phys. Rev. B 40, 7788 (1989); Europhys. Lett. 23, 211 (1993).

${ }^{11}$ The Quantum Hall Effect, edited by R. Prange and S. Girvin (Springer, Berlin, 1990), and references therein. 